\title{
Ancilla-Assisted Discrimination of Quantum Gates
}

\author{
Jianxin Chen and Mingsheng Ying
}

\begin{abstract}
The intrinsic idea of superdense coding is to find as many gates as possible such that they can be perfectly discriminated. In this paper, we consider a new scheme of discrimination of quantum gates, called ancillaassisted discrimination, in which a set of quantum gates on a $d$-dimensional system are perfectly discriminated with assistance from an $r$-dimensional ancilla system. The main contribution of the present paper is two-fold: (1) The number of quantum gates that can be discriminated in this scheme is evaluated. We prove that any $r d+1$ quantum gates cannot be perfectly discriminated with assistance from the ancilla, and there exist $r d$ quantum gates which can be perfectly discriminated with assistance from the ancilla. (2) The dimensionality of the minimal ancilla system is estimated. We prove that there exists a constant positive number $c$ such that for any $k \leq c r$ quantum gates, if they are $d$-assisted discriminable, then they are also $r$-assisted discriminable, and there are $c^{\prime} r\left(c^{\prime}>c\right)$ different quantum gates which can be discriminated with a $d$-dimensional ancilla, but they cannot be discriminated if the ancilla is reduced to an $r$-dimensional system. Thus, the order $O(r)$ of the number of quantum gates that can be discriminated with assistance from an $r$-dimensional ancilla is optimal. The results reported in this paper represent a preliminary step toward understanding the role ancilla system plays in discrimination of quantum gates as well as the power and limit of superdense coding.
\end{abstract}

Index Terms-superdense coding, discrimination, quantum gates, ancilla system, algebraic geometry.

\section{INTRODUCTION}

$\mathbf{H}$ OW to determine the oracle from many known candidates if the investigation into internal structures of oracle is not allowed? This is one of the central questions in theoretical computer science named as oracle identification problem or sometimes circuit verification problem.

In quantum information theory, a special case of the oracle identification problems is the discrimination of quantum states [?]. This is a fundamental task in quantum information theory since information is encoded in states and after accomplishing some quantum information tasks, information encoded in the states has to be read out, that means the final state of the system has to be determined. It is well known that perfect discrimination is possible only for orthogonal quantum states [1].

J. Chen and M. Ying are with the State Key Laboratory of Intelligent Technology and Systems, Tsinghua National Laboratory for Information Science and Technology, Department of Computer Science and Technology, Tsinghua University, Beijing 100084, China. e-mail: chenkenshin@gmail.com (J. Chen), yingmsh@mail.tsinghua.edu.cn (M. Ying)
Thus, so-called unambiguous discrimination strategy is introduced for quantum states which are not necessarily mutually orthogonal [2], [3], [4], [5], [6], [7], [?], [10], [11], [12], [13], [14]. In contrast to perfect discrimination where one can always identify the state, unambiguous discrimination guarantees that except for an inconclusive probability, one can always get the correct state with zero error probability. It was shown that a set of quantum pure states are unambiguously discriminable if and only if they are linearly independent [6]. Furthermore, many variations of state discrimination problem such as local discrimination, discrimination of mixed states, discrimination of multipartite quantum states, minimum-error discrimination and asymptotic discrimination were proposed and have attracted much attention [15], [16], [17], [18], [19], [20], [?], [?], [?], [21], [22]. For a comprehensive survey of state discrimination, we refer to [23], [24].

Another class of the oracle identification problems arisen in quantum information theory is the discrimination of quantum gates which is indeed closely related to the discrimination of quantum states. The problem of discriminating oracle quantum gates can be formally stated as follows. Suppose we are given an unknown quantum gate, namely a unitary operator, which is secretely chosen from a set $\left\{U_{1}, \ldots, U_{k}\right\}$ of quantum gates acting on a quantum system whose state space is a $d$-dimensional Hilbert space $\mathcal{H}_{d}$. The aim is to decide which one is the given gate. The discrimination of $U_{1}, \ldots, U_{k}$ can be realized by transforming it to a corresponding problem of discriminating quantum states, more precisely, by choosing a suitable input state $|\psi\rangle \in \mathcal{H}_{d}$ and then discriminating $U_{1}|\psi\rangle, \ldots, U_{k}|\psi\rangle$.

Several variants of the problem of discriminating quantum operations have been considered in the literature. For example, unambiguous discrimination is introduced to the discrimination of quantum operations [25], [26]. If we consider quantum gates acting on many particles, discrimination using local operations and classical communication is then proposed and has been extensively studied [27], [28], [29].

It should be remarked that, for perfect discrimination of quantum gates, it was shown that any two different quantum gates can always be perfectly discriminated if multicopy is allowed, which is quite different from perfect discrimination of quantum states, where infinite copies are required in order to attain perfect discrimination [30]. Thus the multicopy strategy would greatly extends the ability to discriminate quantum gates. 
In this paper, we consider another possibility of improving ability of discriminating quantum gates, namely discrimination with an ancilla system. If ancilla system is allowed, then the ability of discriminating quantum gates acting on $d$-dimensional Hilbert space should be improved. However, any reasonable ancilla system in practice generally cannot possibly extend to large scale. Thus we assume the dimension of ancilla system is bounded by some $r \leq d$. In this situation, we say these quantum gates can be $r$-assisted discriminated.

The purpose of the present paper is to address the following two questions and to explore the role ancilla system plays in discriminating among quantum oracle gates.

Problem 1. [Maximal Discrimination Problem] How many quantum gates acting on $d$-dimensional Hilbert space at most can be perfectly discriminated with assistance from an $r$-dimensional ancilla?

Problem 2. [r-Reduced Discrimination Problem] What is the maximum number $N$ such that for any $N$ quantum gates acting on $d$-dimensional Hilbert spaces, if they can be perfectly discriminated with some large ancilla system, then they can still be discriminated when the ancilla system is reduced to $r$-dimensional.

Ancilla-assisted discrimination of quantum gates is a fundamental problem in quantum information theory. Its importance is witnessed by the simple observation that the intrinsic idea of superdense coding is to find as many gates as possible such that they can be perfectly discriminated by some state [1], [31]. To be more precise, let's recall the protocol for standard superdense coding. Suppose there are two parties, known as Alice and Bob, who are far away from one another. Alice possesses a $p$ qubit system $\mathcal{A}$ and Bob holds a $q$-qubit system $\mathcal{B}$. Their goal is to transmit as much information as possible. Then Alice can send $k$ bits of classical information to Bob using only $p$ qubits by following procedure if there exist $2^{k}$ quantum gates $\left\{U_{i}\right\}_{i=1}^{2^{k}}$ acting on $\mathcal{A}$ which can be $2^{q}$ assisted discriminable. Let Alice and Bob initially share a state $|\psi\rangle_{\mathcal{A B}}$ which satisfies

$$
\left\langle\left.\psi\right|_{\mathcal{A B}}\left(U_{i} \otimes I_{\mathcal{B}}\right)^{\dagger}\left(U_{j} \otimes I_{\mathcal{B}}\right) \mid \psi\right\rangle_{\mathcal{A B}}=0
$$

for all $1 \leq i \neq j \leq 2^{k}$. The existence of such $|\psi\rangle$ is equivalent to $2^{q}$-assisted discriminability of these quantum gates. If Alice wishes to send the bit string ' ${ }^{\prime} i_{1} i_{2} \cdots i_{k}{ }^{\prime}$ to Bob, she applies the quantum gate $U_{i_{1} i_{2} \cdots i_{k}}$ to her qubits, and then sends her qubits to Bob, giving Bob possession of the whole system. Notice that

$$
\left\langle\left.\psi\right|_{\mathcal{A B}}\left(U_{i} \otimes I_{\mathcal{B}}\right)^{\dagger}\left(U_{j} \otimes I_{\mathcal{B}}\right) \mid \psi\right\rangle_{\mathcal{A B}}=0
$$

for all $1 \leq i \neq j \leq 2^{k}$ is equivalent to $\left\{\left(U_{i} \otimes I_{\mathcal{B}}\right)|\psi\rangle_{\mathcal{A B}}\right\}_{i=1}^{2^{k}}$ form a subset of an orthogonal basis. Thus Bob can do a measurement in this basis, and then determine which of the bit strings Alice sent. Thus the natural strategy of optimal superdense coding scheme is to find out as many unitary gates as possible, which can be perfectly discriminated with assistance from $\mathcal{B}$.

The paper is structured as follows. After introducing our notations and giving some background on discrimination of quantum gates in section [1 we give a straightforward and complete solution to Problem 1 in section $\amalg$. Section III is devoted to answer Problem 2, This section is divided into two subsections. In the first subsection, we present an upper bound for Problem 2, and a lower bound for this problem is given in the second subsection. To prove the theorem concerning the lower bound, some basic ideas from algebraic geometry are required. For convenience of the reader, the necessary concepts and theorems in algebraic geometry are included in the second subsection. Finally, we conclude and present a number of open problems.

Through this paper, we focus on perfectly discrimination strategy. And for simplicity, sometimes we may use the word "discrimination" directly instead of "perfectly discrimination" without explicit explanation.

\section{MAXimal Discrimination Problem}

In this section, we will answer Problem 1 We first give a formal definition of discriminability of quantum gates.

Definition 1. Suppose a $d$-dimensional Hilbert space $\mathcal{H}_{d}$ is given, consider a set $\mathcal{U}=\left\{U_{i}\right\}_{i=1}^{k}$ of quantum gates acting on $\mathcal{H}_{d}$.

1) We say $\mathcal{U}$ is (or $U_{1}, \ldots, U_{k}$ are) discriminable if it (or they) will output a set of orthogonal states for some given initial input state; that is, there exists $|\psi\rangle \in \mathcal{H}_{d}$ such that $\left\langle\psi\left|U_{i}^{\dagger} U_{j}\right| \psi\right\rangle=0$ for all $1 \leq i \neq$ $j \leq k$.

2) We say $\mathcal{U}$ is (or $U_{1}, \ldots, U_{k}$ are) $r$-assisted discriminable if it is (or they are) discriminable with an $r$-dimensional ancilla system $\mathcal{H}_{R}$; that is, there exists a pure state $|\psi\rangle \in \mathcal{H}_{d} \otimes \mathcal{H}_{R}$ such that $\left\langle\psi\left|\left(U_{i} \otimes I_{R}\right)^{\dagger}\left(U_{j} \otimes I_{R}\right)\right| \psi\right\rangle=0$ for all $1 \leq i \neq j \leq k$.

The following two technical lemmas will be needed in the proof of our main theorem in this section.

Lemma 1. $k$ quantum gates $\left\{U_{i}\right\}_{i=1}^{k}$ are r-assisted discriminable if and only if there exists a density operator $\rho$ with rank $\leq r$ such that

$$
\operatorname{tr}\left(U_{i}^{\dagger} U_{j} \rho\right)=0 \text { for all } 1 \leq i \neq j \leq k .
$$

Proof: Assume $\left\{U_{i}\right\}_{i=1}^{k}$ are $r$-assisted discriminable. Then there exists a state $|\psi\rangle \in \mathcal{H}_{d} \otimes \mathcal{H}_{R}$ such that

$$
\left\langle\psi\left|\left(U_{i} \otimes I_{R}\right)^{\dagger}\left(U_{j} \otimes I_{R}\right)\right| \psi\right\rangle=0
$$

for all $1 \leq i \neq j \leq k$. We noticed that any $|\psi\rangle \in \mathcal{H}_{d} \otimes \mathcal{H}_{R}$ can be expressed as $\sum_{l=0}^{r-1}\left|\psi_{l}\right\rangle|l\rangle_{R}$, where $\left\{|l\rangle_{R}\right\}_{l=0}^{r-1}$ form an orthogonal basis of the ancilla system, and $\left|\psi_{l}\right\rangle \in \mathcal{H}_{d}$ are unnormalized states for all $0 \leq l \leq r-1$. It follows 
immediately that

$$
\begin{aligned}
\sum_{l=0}^{r-1} \quad & \left\langle\psi_{l}\left|\left\langle\left. l\right|_{R}\left(U_{i} \otimes I_{R}\right)^{\dagger}\left(U_{j} \otimes I_{R}\right) \sum_{l^{\prime}=0}^{r-1} \mid \psi_{l^{\prime}}\right\rangle\right| l^{\prime}\right\rangle_{R} \\
& =\sum_{l=0}^{r-1}\left\langle\psi_{l}\left|U_{i}^{\dagger} U_{j}\right| \psi_{l}\right\rangle \\
& =\operatorname{tr}\left(U_{i}^{\dagger} U_{j} \sum_{l=0}^{r-1}\left|\psi_{l}\right\rangle\left\langle\psi_{l}\right|\right),
\end{aligned}
$$

So, $\rho=\sum_{l=0}^{r-1}\left|\psi_{l}\right\rangle\left\langle\psi_{l}\right|$ is the required density operator and $\operatorname{rank}(\rho) \leq r$.

Conversely, if a density operator $\rho$ with rank $\leq r$ is given, with $\operatorname{tr}\left(U_{i}^{\dagger} U_{j} \rho\right)=0$ for all $1 \leq i \neq j \leq k$, then we can use the spectrum decomposition to find unnormalized states $\left\{\left|\psi_{l}\right\rangle\right\}_{l=0}^{r-1}$ satisfying $\rho=\sum_{l=0}^{r-1}\left|\psi_{l}\right\rangle\left\langle\psi_{l}\right|$, and further construct the corresponding

$$
|\psi\rangle=\sum_{l=0}^{r-1}\left|\psi_{l}\right\rangle|l\rangle_{R} \in \mathcal{H}_{d} \otimes \mathcal{H}_{R}
$$

It is obvious that such $|\psi\rangle$ satisfies $\left\langle\psi\left|U_{i}^{\dagger} U_{j}\right| \psi\right\rangle=0$ for all $1 \leq i \neq j \leq k$ which means these quantum gates are $r$-assisted discriminable. Thus, we have proved our lemma and sometimes we say that $\left\{U_{i}\right\}_{i=1}^{k}$ are perfectly discriminated by $\rho$.

Recall that a set $\left\{M_{i}\right\}_{i=1}^{k}$ of matrices are said to be mutually orthogonal if $\operatorname{tr}\left(M_{i}^{\dagger} M_{j}\right)=0$ for all $1 \leq i \neq j \leq$ $k$.

Lemma 2. $k$ quantum gates $\left\{U_{i}\right\}_{i=1}^{k}$ acting on $a d$ dimensional Hilbert space are $r$-assisted discriminable if and only if there exists a matrix $S$ with rank $\leq r$ such that $\left\{U_{i} S\right\}_{i=1}^{k}$ are mutually orthogonal; in this case we say that $\left\{U_{i}\right\}_{i=1}^{k}$ are discriminated by $S$.

Proof: From Lemma1, $\left\{U_{i}\right\}_{i=1}^{k}$ are $r$-assisted discriminable if and only if there exists a density operator $\rho$ with rank $\leq r$ such that $\operatorname{tr}\left(U_{i}^{\dagger} U_{j} \rho\right)=0$ for all $1 \leq i \neq j \leq k$. Note that the density operator $\rho$ can be written as $S S^{\dagger}$ for some matrix $S$, and $\operatorname{tr}\left(U_{i}^{\dagger} U_{j} \rho\right)=0$ means $U_{i} S$ is orthogonal to $U_{j} S$. Thus $\left\{U_{i} S\right\}_{i=1}^{k}$ are mutually orthogonal. The converse is also straightforward, and we omit the proof here.

Now we are able to present the main result of this section which give a complete solution to Problem 1.

Theorem 1. If $k$ quantum gates $\left\{U_{i}\right\}_{i=1}^{k}$ acting on $d$ dimensional Hilbert space are $r$-assisted discriminable, then $k \leq r d$, and the upper bound $r d$ can be achieved provided $r \leq d$.

Proof: Suppose $\left\{U_{i}\right\}_{i=1}^{k}$ are $r$-assisted discriminable. Then from Lemma 2 we know that there exists a matrix $S$ with rank $\leq r$ satisfying mutual orthogonality of $\left\{U_{i} S\right\}_{i=1}^{k}$. We can write $S$ in the following way:

$$
S=\sum_{k=1}^{r}\left|p_{k}\right\rangle\left\langle q_{k}\right| \text {. }
$$

Then $U_{i} S$ lies in the subspace $H_{d} \otimes \operatorname{span}\left(\left\{\left\langle q_{j}\right|\right\}_{j=1}^{r}\right)$ whose dimension is at most $r d$. This together with the fact that $\left\{U_{i}\right\}_{i=1}^{k}$ are mutually orthogonal implies $k \leq r d$.

To illustrate our observation, we introduce several elementary unitary gates first. Suppose $\{|k\rangle\}_{k=1}^{d}$ is the computational basis of a qudit space $\mathcal{H}_{d}$. We define $X$ by $X|k\rangle=|k+1\rangle$, where the addition is modulo $d$, and $Z$ by $Z|k\rangle=\omega^{k}|k\rangle$, where $\omega=e^{-\frac{2 \pi i}{d}}$ is the $d$ th unity root. Then the set of the generalized Pauli matrices in the qudit system is $\left\{X^{p} Z^{q}: p, q=0, \cdots, d-1\right\}$.

Now we assume that $r \leq d$. For the simplest case of $r=1$, we choose $d$ gates: $\left\{X^{i}\right\}_{i=0}^{d-1}$. Obviously, they can be discriminated by $\operatorname{diag}\{1,0, \cdots, 0\}$. For the case of $r \geq$ 2 , let $\omega$ be the unity root of order $r$, and let

$$
W=\operatorname{diag}\left\{1, \omega, \omega^{2}, \cdots, \omega^{r-1}, 1, \cdots, 1\right\} .
$$

We choose $r d$ quantum gates: $\left\{X^{i} W^{j}\right\}$, where $0 \leq i \leq$ $r-1,0 \leq j \leq d-1$. It is easy to see that they can be discriminated by $\rho=\operatorname{diag}\left\{\frac{1}{r}, \cdots, \frac{1}{r}, 0, \cdots, 0\right\}$ whose first $r$ entries in the principle diagonal are all $\frac{1}{r}$ and others are all zero. Thus, we complete the proof by Lemma 1

As a simple application of the above theorem, we return back to the superdense coding scenario considered in the Introduction. With Theorem 1, we have

$$
2^{k} \leq \operatorname{dim}(\mathcal{A}) \operatorname{dim}(\mathcal{B})=2^{p} 2^{q}=2^{p+q} .
$$

which follows $k \leq p+q$. Thus if Alice possesses $p$ qubits and Bob possess $q$ qubits, then Alice can transmit at most $p+q$ bits of classical information to Bob using her $p$ qubits of quantum information, this bound can be achieved provided $q \leq p$.

\section{R-REDUCED DISCRIMINATION PROBLEM}

As pointed in the Introduction, this section is entirely devoted to address Problem 2. Suppose a set $\mathcal{U}$ of quantum gates acting on $d$-dimensional Hilbert space can be perfectly discriminated with assistance from an ancilla system. In the real world, sometimes the large ancilla system is not possible in practice and what we can deal with is a small ancilla system. In this section, we assume some quantum gates can be perfectly discriminated with assistance from a large ancilla system, and our goal is to reduce the ancilla such that these quantum gates still can be perfectly discriminated with assistance from the reduced ancilla system.

A simple application of the Schmidt decomposition indicates that the quantum gates acting on $d$-dimensional Hilbert space can always be perfectly discriminated with assistance from a $d$-dimensional ancilla system; in other words, they are always $d$-assisted discriminable. So, it is reasonable to rephrase Problem 2 as the following more specific question:

Problem 3. [r-Reduced Discrimination Problem] Given a positive integer $r<d$. What is the maximum number $N$ satisfying that any $N d$-assisted discriminable quantum gates acting on $d$-dimensional Hilbert space will 
preserve their discriminability when the ancilla system is reduced to dimension $r$.

We will answer this question by showing that, there exist $O(r) d$-assisted discriminable quantum gates acting on $d$-dimensional Hilbert space which cannot be $r$ assisted discriminated. On the other hand, there exists some constant positive number $c$ such that for any $\mathrm{cr}$ quantum gates, if they are perfectly discriminable with assistance from some large ancilla system, then the discriminability remains when the ancilla system is reduced to $r$-dimensional.

\section{A. Upper Bound of r-Reduced Discrimination problem}

In this subsection, we will show the upper bound for Problem 3 in the following three steps.

1. Starting from a simple observation at the ancillafree discrimination.

2. We will derive an upper bound $2 \sqrt{r d}$ for general situation

3. If $r \leq \frac{d}{2}-1$, then our upper bound can be improved to $2 r$.

From above arguments it follows immediately that there always exist $O(r) d$-assisted discriminable quantum gates acting on $d$-dimensional Hilbert space which cannot be $r$-assisted discriminated.

Let's label the diagonals of a $d \times d$ matrix by integers $k$, with $k$ increasing from lower left to upper right. So there are $2 d-1$ diagonals, and the $i$-th diagonal has $d-|d-x|$ entries. It is easy to see that entries of $X^{k}$ are all 1's in the $(d-k)$-th diagonal or $(2 d-k)$-th diagonal, and all 0 's in other diagonals.

Lemma 3. There does not exist a state $|\psi\rangle \in \mathcal{H}_{d}$ such that $\left\langle\psi\left|X Z^{k}\right| \psi\right\rangle=0$ for all $0 \leq k \leq d-1$ and $\left\langle\psi\left|Z^{k}\right| \psi\right\rangle=0$ for all $1 \leq k \leq d-1$.

Proof: If there exists a state $|\psi\rangle \in \mathcal{H}_{d}$ satisfies the equations in the lemma, then it can be written in the computational basis as $|\psi\rangle=\sum_{i=0}^{d-1} \lambda_{i}|i\rangle$, and

$$
\begin{aligned}
\left\langle\psi\left|X Z^{k}\right| \psi\right\rangle=0 & \Longleftrightarrow \quad\left(\sum_{i=0}^{d-1} \lambda_{i}^{*}\langle i|\right) X Z^{k}\left(\sum_{j=0}^{d-1} \lambda_{j}|j\rangle\right)=0 \\
& \Longleftrightarrow \quad \sum_{i, j=0}^{d-1} \lambda_{i}^{*} \lambda_{j}\left\langle i\left|X Z^{k}\right| j\right\rangle=0 \\
& \Longleftrightarrow \quad \sum_{j=0}^{d-1} \omega^{k j} \lambda_{j+1}^{*} \lambda_{j}=0 .
\end{aligned}
$$

Since $\left\langle\psi\left|X Z^{k}\right| \psi\right\rangle=0$ for all $0 \leq k \leq d-1$, we have the following linear equations expressed in the matrix form:

$$
\left(\begin{array}{cccc}
1 & 1 & \cdots & 1 \\
1 & \omega & \cdots & \omega^{d-1} \\
\vdots & \vdots & \ddots & \vdots \\
1 & \omega^{d-1} & \cdots & \omega^{(d-1)(d-1)}
\end{array}\right)\left(\begin{array}{c}
\lambda_{1}^{*} \lambda_{0} \\
\lambda_{2}^{*} \lambda_{1} \\
\vdots \\
\lambda_{d}^{*} \lambda_{d-1}
\end{array}\right)=\mathcal{O},
$$

which implies $\lambda_{i+1}^{*} \lambda_{i}=0$ for all $1 \leq i \leq d$.

On the other hand, $\left\langle\psi\left|Z^{k}\right| \psi\right\rangle=0$ with $1 \leq k \leq d-1$ can be rewritten as the following matrix equation:

$$
\left(\begin{array}{cccc}
1 & \omega & \cdots & \omega^{d-1} \\
1 & \omega^{2} & \cdots & \omega^{2(d-1)} \\
\vdots & \vdots & \ddots & \vdots \\
1 & \omega^{d-1} & \cdots & \omega^{(d-1)(d-1)}
\end{array}\right)\left(\begin{array}{c}
\lambda_{0}^{*} \lambda_{0} \\
\lambda_{1}^{*} \lambda_{1} \\
\vdots \\
\lambda_{d-1}^{*} \lambda_{d-1}
\end{array}\right)=\mathcal{O}
$$

Thus we have $\left|\lambda_{0}\right|=\cdots\left|\lambda_{i}\right|=\cdots\left|\lambda_{d-1}\right|$. Combining this with the previous conclusion $\lambda_{i+1}^{*} \lambda_{i}=0$ for all $1 \leq i \leq d$, we obtain $|\psi\rangle=0$.

Lemma 4. There exist $O(\sqrt{d})$ quantum gates acting on a $d$ dimensional Hilbert space which are d-assisted discriminable, but cannot be perfectly discriminated if the ancilla system is not allowed.

Proof: We choose the following quantum gates:

$$
\begin{array}{cccc}
Z & Z^{2} & \cdots & Z^{\left\lfloor\frac{p}{2}\right\rfloor} \\
I & Z^{p} & \cdots & Z^{\left\lceil\frac{d}{p}\right\rceil p} \\
X & X Z^{p} & \cdots & X Z^{\left\lceil\frac{d}{p}\right\rceil p} \\
X^{d-1} & X^{d-1} Z^{p} & \cdots & X^{d-1} Z^{\left\lceil\frac{d}{p}\right\rceil p}
\end{array}
$$

where $p$ is an integer which will be fixed later. Following the well-known commutation relations of generalized Pauli group if we ignore the phase factor, $\left(X^{d-1} Z^{i p}\right)^{\dagger} Z^{j}$, $\left(Z^{j}\right)^{\dagger} X Z^{i p},\left(Z^{i p}\right)^{\dagger} Z^{j},\left(Z^{j}\right)^{\dagger} Z^{i p}$ can be identified with $X Z^{-i p+j}, X Z^{i p-j}, Z^{-i p+j}$ and $Z^{i p-j}$ respectively.

Now we divide our proof into the following two steps. First, we show that the above gates cannot be discriminated if the ancilla system is not allowed. If not so, then there exists a pure state $|\psi\rangle$ which perfectly discriminates these quantum gates, and we have the following equations:

$$
\begin{aligned}
\left\langle\psi\left|X Z^{-i p+j}\right| \psi\right\rangle & =0 \\
\left\langle\psi\left|X Z^{i p-j}\right| \psi\right\rangle & =0 \\
\left\langle\psi\left|Z^{-i p+j}\right| \psi\right\rangle & =0 \\
\left\langle\psi\left|Z^{i p-j}\right| \psi\right\rangle & =0
\end{aligned}
$$

where $i$ ranges over $\left\{0,1,2, \cdots,\left\lceil\frac{d}{p}\right\rceil\right\}$ and $j$ ranges over $\left\{0,1,2, \cdots,\left\lfloor\frac{p}{2}\right\rfloor\right\}$, but $(i, j) \neq(0,0)$. Note that the set

$$
\left\{X Z^{-i p+j}\right\}_{i, j} \bigcup\left\{X Z^{i p-j}\right\}_{i, j} \bigcup\left\{Z^{-i p+j}\right\}_{i, j} \bigcup\left\{Z^{i p-j}\right\}_{i, j}
$$

contains the gates $\left\{Z^{k}\right\}_{k=1}^{d-1}$ and $\left\{X Z^{k}\right\}_{k=0}^{d-1}$. Then it holds that $\left\langle\psi\left|X Z^{k}\right| \psi\right\rangle=0$ for all $0 \leq k \leq d-1$ and $\left\langle\psi\left|Z^{k}\right| \psi\right\rangle=$ 0 for all $1 \leq k \leq d-1$, which contradicts Lemma 3 .

Second, we estimate the order of the number of the above gates. It is obvious that the number of the gates is $3\left\lceil\frac{d}{p}\right\rceil+3+\left\lfloor\frac{p}{2}\right\rfloor$. Then we can choose $p=\lceil\sqrt{6 d}\rceil$ and derive following inequalities

$$
\begin{aligned}
\sqrt{6 d}+2 & \leq \frac{3 d}{p}+2+\frac{p}{2} \leq 3\left\lceil\frac{d}{p}\right\rceil+3+\left\lfloor\frac{p}{2}\right\rfloor \\
& \leq \frac{3 d}{p}+6+\frac{p}{2} \leq \frac{3 d}{\sqrt{6 d}}+6+\frac{\sqrt{6 d}+1}{2}=\sqrt{6 d}+\frac{13}{2}
\end{aligned}
$$

which completes our proof. 
Next, we will generalize our idea to $r$-dimensional ancilla-assisted case and show that there exist $O(\sqrt{r d})$ $d$-assisted discriminable quantum gates which cannot be perfectly discriminated with assistance from an $r$ dimensional ancilla system.

Lemma 5. There does not exist any density operator $\rho$ with rank $\leq r$ such that

$$
\operatorname{tr}\left(X^{i} Z^{j} \rho\right)=0
$$

for all $1 \leq i \leq r, 0 \leq j \leq d-1$, and

$$
\operatorname{tr}\left(Z^{j} \rho\right)=0
$$

for all $1 \leq j \leq d-1$.

Proof: We prove the lemma by refutation. Suppose there is a density operator $\rho$ satisfying $\operatorname{tr}\left(X^{i} Z^{j} \rho\right)=0$ for all $1 \leq i \leq r, 0 \leq j \leq d-1$, and $\operatorname{tr}\left(Z^{j} \rho\right)=0$ for all $1 \leq j \leq d-1$ and $\operatorname{rank}(\rho) \leq r$. Notice that $\operatorname{tr}\left(X^{i} Z^{j} \rho\right)=0$ is indeed a constraint of the $(d-i)$-th diagonal of $\rho$. We have $d$ linear equations of the $(d-i)$-th diagonal for each $i \in\{1,2, \cdots, r\}$. The corresponding coefficient matrix is the Vandermonde matrix generated by $\left(1, \omega, \cdots, \omega^{d-1}\right)$ which is nonsingular. So the entries in the $(d-i)$-th diagonal of $\rho$ are all zero for all $1 \leq i \leq r$.

On the other hand, the condition that $\operatorname{tr}\left(Z^{j} \rho\right)=0$ for all $1 \leq j \leq d-1$ gives us $d-1$ linear equations of the principle diagonal of $\rho$. The coefficient matrix is a submatrix of a Vandermonde matrix obtained by deleting the row of $(1,1, \cdots, 1)$. Thus, the entries in the principle diagonal of $\rho$ should form a vector which is orthogonal to the conjugate transpose of each row in the coefficient matrix, and these entries should be the same. Since $\rho$ is a density operator, we have $\operatorname{tr}(\rho)=1$, which implies that the entries in the main diagonal of $\rho$ are all nonzero.

Now consider an $(r+1)$ by $(r+1)$ submatrix $M$ of $\rho$ that lies in the rows indexed from 1 to $r+1$ and the columns indexed from 1 to $r+1$ respectively. Clearly, $M$ is upper triangular and all the entries in its main diagonal are nonzero, and thus has nonzero determinant. So $\rho$ has at least one nonzero order- $(r+1)$ minor, and $\operatorname{rank}(\rho) \geq r+1$. This contradicts our assumption that $\operatorname{rank}(\rho) \leq r$.

Lemma 6. There exist $O(\sqrt{r d})$ quantum gates which are $d$ assisted discriminable but are not r-assisted discriminable.

Proof: Let $p$ and $q$ be two positive integers which will be specified later. We choose $\left\{U_{i}\right\}_{i=1}^{k}$ to be the following set of gates:

$\left\{X^{i} Z^{j}\right\}_{0 \leq i \leq p-1,0 \leq j \leq q-1} \bigcup\left\{X^{-p i^{\prime}} Z^{-q j^{\prime}}\right\}_{0 \leq i^{\prime} \leq\left\lceil\frac{r}{p}\right\rceil, 0 \leq j^{\prime} \leq\left\lceil\frac{d}{q}\right\rceil}$, where these gates are arbitrarily numbered from $U_{1}$ to $U_{k}$, and $k$ is the cardinality of the above set of gates. Then the set $\left\{U_{i}^{\dagger} U_{j}\right\}_{1 \leq i \neq j \leq k}$ contains the following gates:

$$
\left(X^{-p i^{\prime}} Z^{-q j^{\prime}}\right)^{\dagger} X^{i} Z^{j}
$$

where $0 \leq i \leq p-1,0 \leq j \leq q-1,0 \leq i^{\prime} \leq$ $\left\lceil\frac{r}{p}\right\rceil, 0 \leq j^{\prime} \leq\left\lceil\frac{d}{q}\right\rceil$ and $\left(i, j, i^{\prime}, j^{\prime}\right) \neq(0,0,0,0)$. Since $X$ and $Z$ are commutative, the gate $\left(X^{-p i^{\prime}} Z^{-q j^{\prime}}\right)^{\dagger} X^{i} Z^{j}$ can be identified with $X^{p i^{\prime}+i} Z^{q j^{\prime}+j}$ if we ignore the global phase. It is easy to see that the set

$$
\left\{X^{p i^{\prime}+i} Z^{q j^{\prime}+j}\right\}_{i, i, j, j^{\prime}}
$$

with $0 \leq i \leq p-1,0 \leq j \leq q-1,0 \leq i^{\prime} \leq\left\lceil\frac{r}{p}\right\rceil, 0 \leq$ $j^{\prime} \leq\left\lceil\frac{d}{q}\right\rceil$ and $\left(i, j, i^{\prime}, j^{\prime}\right) \neq(0,0,0,0)$, contains all $X^{i} Z^{j}$ for $0 \leq i \leq r, 0 \leq j \leq d-1$ and $(i, j) \neq(0,0)$. Thus, using Lemma 5 we conclude that there is not any density operator with rank $\leq r$ satisfying $\operatorname{tr}\left(X^{i} Z^{j} \rho\right)=0$ for all $1 \leq i \leq r, 0 \leq j \leq d-1$ and $\operatorname{tr}\left(Z^{j} \rho\right)=0$ for all $1 \leq$ $k \leq d-1$. Furthermore, with Lemma 1 we see that these quantum gates cannot be discriminated perfectly with an $r$-dimensional ancilla system.

Now we can set $p$ and $q$ to be $\lceil\sqrt{r}\rceil$ and $\lceil\sqrt{d}\rceil$ respectively. Then the number of quantum gates here is $N=p q+\left(\left\lceil\frac{r}{p}\right\rceil+1\right)\left(\left\lceil\frac{d}{q}\right\rceil+1\right)-1$ which satisfies

$$
2 \sqrt{r d} \leq N \leq 2(\sqrt{r}+2)(\sqrt{d}+2)-1 .
$$

The upper bound given in the above lemma can be improved in some special cases. Suppose $r \ll d$, or more specifically, $r \leq \frac{d}{2}-1$. Then we have the following lemma which tightens our previous upper bound significantly.

Lemma 7. Let $r \leq \frac{d}{2}-1$. Then there are $2 r+2$ quantum gates which can be d-assisted discriminated but cannot be r-assisted discriminated.

Proof: We assume $r$ is even, let $r=2 r_{1}$. Let $\alpha$ be the $(r+1)$-th unity root and $\beta$ be the $(d-r-1)$-th unity root respectively. Choose following quantum gates as $\mathcal{U}=$ $\left\{U_{i}\right\}_{i=1}^{k}$ :

$$
\begin{array}{cl}
X_{r+1}^{i} \bigoplus X_{d-r-1}^{i} & 1 \leq i \leq r_{1} \\
X_{r+1}^{-i} \bigoplus \omega X_{d-r-1}^{-i} & 1 \leq i \leq r_{1} \\
Z_{r+1}^{j} \bigoplus Z_{d-r-1}^{j} & -r_{1} \leq j \leq r_{1} \\
I_{r+1} \bigoplus(-1) I_{d-r-1} &
\end{array}
$$

Here $\omega$ is some unity root. We can assume it is $p$-th unity root where $p$ is a very large prime number.

It is not difficult to check that these quantum gates can be perfectly discriminated by

$$
\frac{1}{2(r+1)} I_{r+1} \bigoplus \frac{1}{2(d-r-1)} I_{d-r-1},
$$

thus can be perfectly discriminated with assistance from a $d$-dimensional ancilla system.

Next, we show that there does not exist a density operator $\rho$ with $\operatorname{rank}(\rho) \leq r$ satisfying following equations:

$$
\operatorname{tr}\left(U_{i}^{\dagger} U_{j} \rho\right)=0 \text { for all } 1 \leq i \neq j \leq|\mathcal{U}|
$$

This will be done by refutation. Suppose there exists a density operator $\rho$ satisfying the above equations. Then 
we have the following equations:

$$
\begin{array}{r}
\operatorname{tr}\left(X_{r+1}^{-i} Z_{r+1}^{j} \bigoplus X_{d-r-1}^{-i} Z_{d-r-1}^{j} \rho\right)=0 \\
\quad \text { or all } 1 \leq i \leq r_{1}, \quad-r_{1} \leq j \leq r_{1} \\
\operatorname{tr}\left(X_{r+1}^{i} Z_{r+1}^{j} \bigoplus \omega^{*} X_{d-r-1}^{i} Z_{d-r-1}^{j} \rho\right)=0 \\
\quad \text { for all } 1 \leq i \leq r_{1}, \quad-r_{1} \leq j \leq r_{1} \\
\operatorname{tr}\left(Z_{r+1}^{-j} X_{r+1}^{i} \bigoplus Z_{d-r-1}^{-j} X_{d-r-1}^{i} \rho\right)=0 \\
\quad \text { for all } 1 \leq i \leq r_{1}, \quad-r_{1} \leq j \leq r_{1} \\
\operatorname{tr}\left(Z_{r+1}^{-j} X_{r+1}^{-i} \bigoplus \omega Z_{d-r-1}^{-j} X_{d-r-1}^{-i} \rho\right)=0 \\
\quad \text { for all } 1 \leq i \leq r_{1}, \quad-r_{1} \leq j \leq r_{1}
\end{array}
$$

Let's replace the index $j$ by $-j$. Notice that

$$
Z_{k}^{q} X_{k}^{p}=\omega_{k}^{p q} X_{k}^{p} Z_{k}^{q}
$$

where $\omega_{k}$ is the $k$-th unity root. Recalling that $\omega_{r+1}=\alpha$ and $\omega_{d-r-1}=\beta$, we obtain:

$$
\begin{array}{r}
\operatorname{tr}\left(X_{r+1}^{-i} Z_{r+1}^{j} \bigoplus X_{d-r-1}^{-i} Z_{d-r-1}^{j} \rho\right)=0 \\
\text { for all } 1 \leq i \leq r_{1}, \quad-r_{1} \leq j \leq r_{1} \\
\operatorname{tr}\left(X_{r+1}^{i} Z_{r+1}^{j} \bigoplus\left(\omega^{*}\right) X_{d-r-1}^{i} Z_{d-r-1}^{j} \rho\right)=0 \\
\text { for all } 1 \leq i \leq r_{1}, \quad-r_{1} \leq j \leq r_{1} \\
\operatorname{tr}\left(\alpha^{i j} X_{r+1}^{i} Z_{r+1}^{j} \bigoplus \beta^{i j} X_{d-r-1}^{i} Z_{d-r-1}^{j} \rho\right)=0 \\
\quad \text { or all } 1 \leq i \leq r_{1}, \quad-r_{1} \leq j \leq r_{1} \\
\operatorname{tr}\left(\alpha^{-i j} X_{r+1}^{-i} Z_{r+1}^{j} \bigoplus \omega \beta^{-i j} X_{d-r-1}^{-i} Z_{d-r-1}^{j} \rho\right)=0 \\
\text { for all } 1 \leq i \leq r_{1}, \quad-r_{1} \leq j \leq r_{1}
\end{array}
$$

Thus, it is straightforward to derive that

$$
\begin{array}{r}
\operatorname{tr}\left(X_{r+1}^{-i} Z_{r+1}^{j} \bigoplus O_{d-r-1} \rho\right)=0 \\
\text { for all } 1 \leq i \leq r_{1}, \quad-r_{1} \leq j \leq r_{1} \\
\quad \operatorname{tr}\left(X_{r+1}^{i} Z_{r+1}^{j} \bigoplus O_{d-r-1} \rho\right)=0 \\
\text { for all } 1 \leq i \leq r_{1}, \quad-r_{1} \leq j \leq r_{1}
\end{array}
$$

These equations describe the constraints on the left-top $(r+1) \times(r+1)$ submatrix $\rho_{0}$ of $\rho$. Furthermore, except for the principle diagonal, each diagonal of $\rho_{0}$ is a solution of these $(r+1)$ linear equations, and the coefficients of these equations form a Vandermonde matrix generated by $(r+1)$-th unity roots. Thus the entries in these diagonals are all zero.

Next, consider the principle diagonal of $\rho$ :

$$
\begin{gathered}
Z_{r+1}^{j} \bigoplus Z_{d-r-1}^{j} \quad-r_{1} \leq j \leq r_{1} \\
I_{r+1} \bigoplus(-1) I_{d-r-1}
\end{gathered}
$$

We have:

$$
\begin{aligned}
\operatorname{tr}\left(Z_{r+1}^{j} \bigoplus Z_{d-r-1}^{j} \rho\right)=0 & \text { for all }-r_{1} \leq j(\neq 0) \leq r_{1} \\
\operatorname{tr}\left(Z_{r+1}^{j} \bigoplus-Z_{d-r-1}^{j} \rho\right)=0 & \text { for all }-r_{1} \leq j(\neq 0) \leq r_{1}
\end{aligned}
$$

Thus,

$\operatorname{tr}\left(Z_{r+1}^{j} \bigoplus O_{d-r-1} \rho\right)=0 \quad$ for all $-r_{1} \leq j(\neq 0) \leq r_{1}$

which suggests the diagonal entries in $\rho_{0}$ are all the same. On the other hand,

$$
\operatorname{tr}\left(I_{r+1} \bigoplus(-1) I_{d-r-1} \rho\right)=0
$$

implies

$$
\sum_{i=1}^{r+1} \rho_{i i}=\sum_{i=r+2}^{d} \rho_{i i}
$$

Since $\rho$ is positive, all entries in the principle diagonal of $\rho$ are nonnegative. Thus, we see that $\rho_{11}$ is non zero. Otherwise all entries in the principle diagonal of $\rho$ are all zero, which contradicts $\operatorname{tr}(\rho) \neq 0$.

The top-left $(r+1) \times(r+1)$ submatrix of $\rho$ is a diagonal matrix whose principle entries are all nonzero. Thus this $(r+1) \times(r+1)$ submatrix is nonsingular, which implies a nonzero minor, and follows by $\operatorname{rank}(\rho) \geq r+1$. This is a contradiction to our assumption. Thus these quantum gates cannot be perfectly discriminated with assistance from an $r$-dimensional ancilla system.

For the case that $r$ is odd, above arguments still hold with a minor modification.

Combining Lemmas 6 and 7 we achieve the main result of this subsection:

Theorem 2. For any $r<d$, we can find $O(r)$ quantum gates which can be d-assisted discriminated but cannot be r-assisted discriminated.

Proof: We consider the following two cases:

Case 1. $r \geq \frac{d}{2}$. From Lemma 6 , we have $O(\sqrt{r d})$ quantum gates which can be $d$-assisted discriminated but cannot be $r$-assisted discriminated. More specifically, for such $r$, we can always find no more than $c \sqrt{r d}$ quantum gates which can be $d$-assisted discriminated but cannot be $r$-assisted discriminated where $c$ is some constant positive number. Since $d \leq 2 r$, we have $c \sqrt{r d} \leq c \sqrt{2} r$. Put $c^{\prime}=c \sqrt{2}$. Then we can always find no more than $c^{\prime} r$ quantum gates which can be $d$-assisted discrimination but cannot be $r$-assisted discrimination.

Case 2. $r \leq \frac{d}{2}-1$. We only need $2 r+2$ quantum gates by Lemma 7

\section{B. Lower Bound of r-Reduced Problem}

We first state the main result of this subsection which presents a lower bound for Problem 3.

Theorem 3. Any

$$
k \leq\left\lfloor\frac{\sqrt{3(r-1)^{2}-19}+1}{2}\right\rfloor
$$

quantum gates which are d-assisted discriminable must also be $r$-assisted discriminable.

The proof of the above requires certain mathematical tools from algebraic geometry. For convenience of the reader, we first recall some definitions and results in algebraic geometry. For details, we refer to [32], [33], [34].

Let $\mathbb{A}^{n}$ be an affine $n$-space, the set of all $n$-tuples of complex numbers. We write $\mathbb{C}\left[x_{1}, x_{2}, \cdots, x_{n}\right]$ for the polynomial ring in $n$ variables. A subset of $A^{n}$ is an algebraic set or algebraic variety if it is the common zeros of a finite set of polynomials $f_{1}, f_{2}, \cdots, f_{r}$ with $f_{i} \in$ $\mathbb{C}\left[x_{1}, x_{2}, \cdots, x_{n}\right]$ for all $1 \leq i \leq r$. Such an algebraic set 
is usually denoted by $Z\left(f_{1}, f_{2}, \cdots, f_{r}\right)$. It is not hard to check that the union of a finite number of algebraic sets is an algebraic set, and the intersection of any family of algebraic sets is again an algebraic set. Thus by taking the open subsets to be the complements of algebraic sets, we can define a topology, called the Zariski topology on $\mathbb{A}^{n}$. Notice that in some references an algebraic variety should also be irreducible in the sense that it cannot be expressed as the union of two proper algebraic sets.

Suppose we can write $\mathcal{X}=\bigcup X_{i}$, as a finite union of irreducible closed sets. If $X_{i} \nsubseteq X_{j}$ for all $i \neq j$, we say such a representation is irredundant, and the $X_{i}$ are irreducible components of $X$.

We define projective $n$-space, denoted by $\mathbb{P}^{n}$, to be the set of equivalence classes of $(n+1)$-tuples $\left(a_{0}, \cdots, a_{n}\right)$ of complex numbers, not all zero, under the equivalence relation given by $\left(a_{0}, \cdots, a_{n}\right) \sim\left(\lambda a_{0}, \cdots, \lambda a_{n}\right)$ for all $\lambda \in \mathbb{C}, \lambda \neq 0$.

A notion of algebraic variety may also be introduced in projective spaces, called projective algebraic variety: a subset $Y$ of $\mathbb{P}^{n}$ is an algebraic set if it is the common zeros of a finite set of homogeneous polynomials $f_{1}, f_{2}, \cdots, f_{r}$ with $f_{i} \in \mathbb{C}\left[x_{0}, x_{1}, \cdots, x_{n}\right]$ for $1 \leq i \leq r$. We call open subsets of irreducible projective varieties as quasiprojective varieties.

Lemma 8 ([34], Chapter 1, Section 6.2, Corollary 5). The variety of common zeros of $r$ polynomials $f_{1}, \cdots, f_{r}$ on an $n$-dimensional projective variety has dimension $\geq n-r$.

Theorem 4 ([34], Chapter 1, Section 6.2, Theorem 6). Let $X, Y \subset \mathbb{P}^{N}$ be irreducible quasiprojective varieties with $\operatorname{dim}(X)=n$ and $\operatorname{dim}(Y)=m$. Then any (nonempty) component $Z$ of $X \cap Y$ has $\operatorname{dim}(Z) \geq n+m-N$. Moreover, if $X$ and $Y$ are projective and $n+m \geq N$ then $X \cap Y \neq \emptyset$.

One may notice that a matrix $M$ has rank no more than $r$ if and only if all the determinants of its $(r+1) \times(r+1)$ submatrices are zero. Thus all $d_{A} \times d_{B}$ matrices with rank $\leq r$ can be considered as a variety, namely the determinantal variety, whose projective dimension is $d_{A} d_{B}-\left(d_{A}-r\right)\left(d_{B}-r\right)-1$. Some applications of the determinantal variety in quantum information theory has been found recently [35], [36]. For more details, we refer to [37.

Proof of Theorem 3 . From Lemma 1] the theorem is equivalent to the following:

Claim: For any

$$
k \leq\left\lfloor\frac{\sqrt{3(r-1)^{2}-19}+1}{2}\right\rfloor
$$

quantum gates $\left\{U_{i}\right\}_{i=1}^{k}$, if there is a density operator $\rho_{0}$, with $\operatorname{rank}\left(\rho_{0}\right)=d$ and $\operatorname{tr}\left(U_{i}^{\dagger} U_{j} \rho_{0}\right)=0$ for all $1 \leq i \neq$ $j \leq k$, then we can always find another density operator $\rho$ satisfying $\operatorname{tr}\left(U_{i}^{\dagger} U_{j} \rho\right)=0$ for all $1 \leq i \neq j \leq k$ and its rank is bounded by $r$.

We will prove the above claim by applying a dimension reduction technique:
First, the base case is $r=d$, which is quite straightforward. Since these quantum gates are $d$-assisted discriminable, we can always find a density operator $\rho$ satisfying these equations, thus it is true for $r=d$.

Second, suppose for any $r$ satisfies $t \leq r \leq d$, the claim is true, then consider the case $r=t-1$. For any given

$$
k \leq\left\lfloor\frac{\sqrt{3(r-1)^{2}-19}+1}{2}\right\rfloor=\left\lfloor\frac{\sqrt{3(t-2)^{2}-19}+1}{2}\right\rfloor
$$

quantum gates which can be perfectly discriminated with assistance from a $d$-dimensional ancilla system, we will show that there exists a density operator $\rho$ with $\operatorname{rank}(\rho) \leq t-1$ such that $\operatorname{tr}\left(U_{i}^{\dagger} U_{j} \rho\right)=0$ for all $1 \leq i \neq j \leq k$. Because

$$
k \leq\left\lfloor\frac{\sqrt{3(t-2)^{2}-19}+1}{2}\right\rfloor \leq\left\lfloor\frac{\sqrt{3(t-1)^{2}-19}+1}{2}\right\rfloor,
$$

there exists a density operator $\rho$ with $\operatorname{rank}(\rho) \leq t$ such that $\operatorname{tr}\left(U_{i}^{\dagger} U_{j} \rho\right)=0$ for all $1 \leq i \neq j \leq k$ from our assumption. If $\operatorname{rank}(\rho) \leq r=t-1$, then the claim is obviously true for $r=t-1$. Otherwise, we assume $\operatorname{rank}(\rho)=t$. Without loss of generality, suppose $\rho=\sum_{i=1}^{t} \lambda_{i}|i\rangle\langle i|$. Then we consider the following two sets:

$$
\mathcal{A}=\left\{X \in M_{t} \mid \operatorname{tr}\left(U_{i}^{\dagger} U_{j} X\right)=0 \forall 1 \leq i \neq j \leq k\right\}
$$

and

$$
\mathcal{B}=\left\{Y \in M_{t} \mid \operatorname{rank}(Y) \leq\left\lfloor\frac{t-1}{2}\right\rfloor\right\}
$$

Here $M_{t}$ is the set of $d \times d$ matrices whose entries are nonzero only in the top left $t \times t$ submatrices. Then $\mathcal{A}$ is a hyperplane of projective dimension at least $t^{2}-1-$ $k(k-1)$, and $\mathcal{B}$ is a determinantal variety of projective dimension $2 t\left\lfloor\frac{t-1}{2}\right\rfloor-\left\lfloor\frac{t-1}{2}\right\rfloor^{2}-1$. Consequently, we have:

$$
\begin{aligned}
& \operatorname{dim}(\mathcal{A})+\operatorname{dim}(\mathcal{B}) \\
\geq & t^{2}-1-k(k-1)+2 t\left\lfloor\frac{t-1}{2}\right\rfloor-\left\lfloor\frac{t-1}{2}\right\rfloor^{2}-1 \\
> & t^{2}-1-k(k-1)-1+2 t\left(\frac{t-3}{2}\right)-\left(\frac{t-3}{2}\right)^{2}-1 \\
> & t^{2}-1 .
\end{aligned}
$$

So, $\mathcal{A} \cap \mathcal{B} \neq \emptyset$ has a component with dimension at least 1 , we can find a nonzero matrix $H_{0} \in \mathcal{A} \cap \mathcal{B}$. It holds that $\operatorname{rank}\left(H_{0}\right) \leq \frac{t-1}{2}$ and $\operatorname{tr}\left(U_{i}^{\dagger} U_{j} H_{0}\right)=0$ for all $1 \leq i \neq$ $j \leq k$. Notice that $\operatorname{tr}\left(\left(U_{i}^{\dagger} U_{j} H_{0}\right)^{\dagger}\right)=0$ and then

$$
\operatorname{tr}\left(U_{j}^{\dagger} U_{i} H_{0}^{\dagger}\right)=\operatorname{tr}\left(H_{0}^{\dagger} U_{j}^{\dagger} U_{i}\right)=0 .
$$

Therefore, we have $\operatorname{tr}\left(U_{i}^{\dagger} U_{j} H_{0}^{\dagger}\right)=0$ for all $1 \leq i \neq j \leq k$, which means $H_{0}^{\dagger} \in \mathcal{A} \cap \mathcal{B}$, and it follows that $H_{0}+H_{0}^{\dagger}$ and $i\left(H_{0}-H_{0}^{\dagger}\right)$ cannot simultaneously be zero matrices. Without loss of generality, suppose $H=H_{0}+H_{0}^{\dagger}$ is not zero, we have $\operatorname{rank}(H) \leq t-1=r$ and $\operatorname{tr}\left(U_{i}^{\dagger} U_{j} H\right)=0$ for all $1 \leq i \neq j \leq k$. Notice the entries in Hermitian matrix $\rho-\lambda H$ are nonzero only in the top left $t \times t$ submatrix. Let $\rho^{\prime}$ and $H^{\prime}$ be the top-left $t \times t$ submatrices 
of $\rho$ and $H$ respectively. We will show that there exists $\lambda$ satisfying

$$
\min _{\left|\psi^{\prime}\right\rangle}\left\langle\psi^{\prime}\left|\rho^{\prime}-\lambda H^{\prime}\right| \psi^{\prime}\right\rangle=0,
$$

which implies

$$
\min _{|\psi\rangle}\langle\psi|\rho-\lambda H| \psi\rangle=0 .
$$

Since we can write $\rho^{\prime}=A^{\dagger} A$, where $A$ is a nonsingular matrix, it holds that

$$
\begin{array}{cl} 
& \min _{\left|\psi^{\prime}\right\rangle}\left\langle\psi^{\prime}\left|\rho^{\prime}-\lambda H^{\prime}\right| \psi^{\prime}\right\rangle=0 \\
\Longleftrightarrow & \min _{\left|\psi^{\prime}\right\rangle}\left\langle\psi^{\prime}\left|A^{\dagger} A-\lambda H^{\prime}\right| \psi^{\prime}\right\rangle=0 \\
\left|\psi^{1}\right\rangle=A\left|\psi^{\prime}\right\rangle & \min _{\left|\psi^{1}\right\rangle}\left\langle\psi^{1}\left|I-\lambda\left(A^{-1}\right)^{\dagger} H^{\prime} A^{-1}\right| \psi^{1}\right\rangle=0 .
\end{array}
$$

Since $\left(A^{-1)^{\dagger}} H A^{-1}\right.$ is Hermitian, it can be expressed as $U^{\dagger} D U$ where $U$ is unitary and $D$ is diagonal. Thus

$$
\begin{array}{ll}
\min _{\left|\psi^{1}\right\rangle}\left\langle\psi^{1}\left|I-\lambda U^{\dagger} D U\right| \psi^{1}\right\rangle=0 \\
\left|\psi^{2}\right\rangle=U\left|\psi^{1}\right\rangle & \min _{\left|\psi^{2}\right\rangle}\left\langle\psi^{2}|I-\lambda D| \psi^{2}\right\rangle=0 .
\end{array}
$$

The existence of $\lambda$ that satisfies the last equation is quite obvious now. Since $D$ is diagonal, we can express it by its entries in the principle diagonal. Assume $D=$ $\operatorname{diag}\left\{d_{1}, d_{2}, \cdots, d_{t}\right\}$ where $d_{1} \geq d_{2} \geq \cdots \geq d_{t}$. If $d_{1}>0$, we can choose $\lambda=\frac{1}{d_{1}}$ satisfying this equation. Otherwise $d_{1} \leq 0$ which means $d_{t} \leq d_{t-1} \leq \cdots \leq d_{1} \leq 0$, and thus $d_{t}<0$. We choose $\lambda=\frac{1}{d_{t}}$ which also satisfies our requirement.

Now we see that there exists $\lambda$ satisfying

$$
\min _{\left|\psi^{\prime}\right\rangle}\left\langle\psi^{\prime}\left|\rho^{\prime}-\lambda H^{\prime}\right| \psi^{\prime}\right\rangle=0,
$$

and then

$$
\min _{|\psi\rangle}\langle\psi|\rho-\lambda H| \psi\rangle=0 .
$$

So, $\rho-\lambda H$ is positive, and $\operatorname{rank}(\rho-\lambda H) \leq t-1$. Both $\rho$ and $H$ are in the linear subspace $\mathcal{A}$, thus so is $\rho-\lambda H$. This implies $\operatorname{tr}\left(U_{i}^{\dagger} U_{j} \rho\right)=0$ for all $1 \leq i \neq j \leq k$. Moreover,

$$
\frac{\rho-\lambda H}{\operatorname{tr}(\rho-\lambda H)}
$$

is the density operator with rank bounded by $t-1$ which can perfectly discriminate these $\left\{U_{i}\right\}_{i=1}^{k}$. So the claim is proved for $r=t-1$. Hence, we proved that the claim is true for any $2 \leq r \leq d$ by reduction on dimension and complete the proof of the theorem.

Remark 5. It should be noted that above argument fails if we reduce the dimension $r$ from 2 to 1 since

$$
\mathcal{B}=\left\{Y \in M_{r} \mid \operatorname{rank}(Y) \leq\left\lfloor\frac{t-1}{2}\right\rfloor=0\right\}
$$

only contains zero matrix. But this doesn't affect our argument very much because we can choose a proper constant coefficient $c$ to make the lower bound hold for all $r$.
Theorem 3 can be restated as the following:

Corollary 6. There exists a constant positive number $c$ such that for any $k \leq \mathrm{cr}$ quantum gates, if they are $d$-assisted discriminable, then they are also r-assisted discriminable.

By comparing the above corollary with Theorem 2 it can be seen that there is indeed no gap between the lower bound and the upper bound. Thus, this corollary together with Theorem 2 completely answers Problem 3.

\section{CONCLUSIONS}

In this paper, we addressed the problem of ancillaassisted perfectly discrimination of quantum gates. We evaluated the number of quantum gates which can be perfectly discriminated with an $r$-dimensional ancilla system. More specifically, we answered following two questions.

1 How many quantum gates at most can be perfectly discriminated?

2 Suppose a set of quantum gates can be perfectly discriminated with some ancilla system. Whether their discriminability remains if the ancilla system is reduced to a small dimension?

For the first question, it is quite simple to observe that any $r d+1$ quantum gates on a $d$-dimensional system cannot be perfectly discriminated with assistance from an $r$-dimensional ancilla. And we showed that there does exist $r d$ quantum gates on a $d$-dimensional system which can be perfectly discriminated with assistance from an $r$-dimensional ancilla.

Our results concerning the second question can be summarized as follows: (1) There exists a constant positive number $c$ such that for any $k \leq c r$ quantum gates on a $d$-dimensional system, if they are $d$-assisted discriminable, then they are also $r$-assisted discriminable. (2) $O(r)$ is optimal. More precisely, there are $c^{\prime} r\left(c^{\prime}>c\right)$ different quantum gates on a $d$-dimensional system which can be $d$-assisted discriminated, but they cannot be perfectly discriminated if the ancilla is reduced to $r$ dimensional.

Discrimination problem is of widely interest because it has many surprising applications. This paper represents a preliminary step toward understanding the role ancilla system plays in discrimination of quantum gates. There are many open questions from this approach deserving further investigation. For example, we may combine our model with the multi-shot discrimination or generalize the input state to several input states as candidates.

\section{ACKNOWLEDGMENT}

We are thankful to the colleagues in the Quantum Computation and Information Research Group of Tsinghua University for helpful discussions. And J. Chen thanks N. Yu for bringing the original question to our attention. J. Chen also thanks J. Yu, Z. Ji for helpful discussion. This work was partly supported by the Natural Science Foundation of China (Grant No. 
60736011, 60621062, 60503001) and the Hi-Tech Research and Development Program of China(863 project)(Grant No. 2006AA01Z102).

\section{REFERENCES}

[1] C. H. Bennett and S. J. Wiesner, "Communication via one- and two-particle operators on einstein-podolsky-rosen states," Phys. Rev. Lett., vol. 69, no. 20, pp. 2881-2884, Nov 1992.

[2] I. D. Ivanovic, "How to differentiate between non-orthogonal states," Phys. Lett. A, vol. 123, no. 6, pp. 257-259, 1987.

[3] I. D. Dieks, "Overlap and distinguishability of quantum states," Phys. Lett. A, vol. 126, no. 5-6, pp. 303-306, 1988.

[4] A. Peres, "How to differentiate between non-orthogonal states," Phys. Lett. A, vol. 128, no. 1-2, p. 19, 1988.

[5] G. Jaeger, "Optimal distinction between two non-orthogonal quantum states," Phys. Lett. A, vol. 197, no. 2, pp. 83-87, 1995.

[6] A. Chefles, "Unambiguous discrimination between linearly independent quantum states," Phys. Lett. A, vol. 239, no. 6, pp. 339 347, 1998.

[7] _ " "Unambiguous discrimination between linearly dependent states with multiple copies," Phys. Rev. A, vol. 64, no. 6, p. 062305, Nov 2001.

[8] X. M. Sun, S. Y. Zhang, Y. Feng, and M. S. Ying, "Mathematical nature of and a family of lower bounds for the success probability of unambiguous discrimination," Phys. Rev. A, vol. 65, no. 4, p. 044306, Apr 2002.

[9] Y. Eldar, "A semidefinite programming approach to optimal unambiguous discrimination of quantum states," Information Theory, IEEE Transactions on, vol. 49, no. 2, pp. 446-456, Feb 2003.

[10] S. Y. Zhang, Y. Feng, X. M. Sun, and M. S. Ying, "Upper bound for the success probability of unambiguous discrimination among quantum states," Phys. Rev. A, vol. 64, no. 6, p. 062103, Nov 2001.

[11] Y.-X. Chen and D. Yang, "Optimal conclusive discrimination of two nonorthogonal pure product multipartite states through local operations," Phys. Rev. A, vol. 64, no. 6, p. 064303, Nov 2001.

[12] Y. Feng, S. Y. Zhang, R. Y. Duan, and M. S. Ying, "Lower bound on inconclusive probability of unambiguous discrimination," Phys. Rev. A, vol. 66, no. 6, p. 062313, Dec 2002.

[13] Y. Feng, R. Y. Duan, and M. S. Ying, "Unambiguous discrimination between mixed quantum states," Phys. Rev. A, vol. 70, no. 1, p. 012308, Jul 2004

[14] M. A. Jafarizadeh, M. Rezaei, N. Karimi, and A. R. Amiri, "Optimal unambiguous discrimination of quantum states," Physical Review A (Atomic, Molecular, and Optical Physics), vol. 77, no. 4, p. 042314, 2008

[15] J. Walgate, A. J. Short, L. Hardy, and V. Vedral, "Local distinguishability of multipartite orthogonal quantum states," Phys. Rev. Lett., vol. 85, no. 23, pp. 4972-4975, Dec 2000.

[16] R. Y. Duan, Y. Feng, Z. F. Ji, and M. S. Ying, "Distinguishing arbitrary multipartite basis unambiguously using local operations and classical communication," Physical Review Letters, vol. 98, no. 23, p. 230502, 2007.

[17] Y. Xin and R. Y. Duan, "Local distinguishability of orthogonal $2 \otimes 3$ pure states," Physical Review A (Atomic, Molecular, and Optical Physics), vol. 77, no. 1, p. 012315, 2008.

[18] T. Rudolph, R. W. Spekkens, and P. S. Turner, "Unambiguous discrimination of mixed states," Phys. Rev. A, vol. 68, no. 1, p. 010301, Jul 2003.

[19] M. Takeoka, M. Ban, and M. Sasaki, "Unambiguous quantumstate filtering," Phys. Rev. A, vol. 68, no. 1, p. 012307, Jul 2003.

[20] P. Raynal, N. Lütkenhaus, and S. J. van Enk, "Reduction theorems for optimal unambiguous state discrimination of density matrices," Phys. Rev. A, vol. 68, no. 2, p. 022308, Aug 2003.

[21] C. Schmid, N. Kiesel, W. Laskowski, W. Wieczorek, M. Żukowski, and H. Weinfurter, "Discriminating multipartite entangled states," Physical Review Letters, vol. 100, no. 20, p. 200407, 2008.

[22] D. Markham, J. A. Miszczak, Z. Puchał a, and K. Życzkowski, "Quantum state discrimination: A geometric approach," Physical Review A (Atomic, Molecular, and Optical Physics), vol. 77, no. 4, p. 042111, 2008.

[23] C. A., "Quantum state discrimination," Contemporary Physics, vol. 41, pp. 401-424(24), 1 November 2000.

[24] M. Paris and J. Rehacek, Eds., Quantum State Estimation. Springer Berlin / Heidelberg, 2004, vol. 649.
[25] A. Chefles, A. Kitagawa, M. Takeoka, M. Sasaki, and J. Twamley, "Unambiguous discrimination among oracle operators," J. Phys. A: Math. Theor., vol. 40, pp. 10183-10 213, 2007.

[26] G. M. Wang and M. S. Ying, "Unambiguous discrimination among quantum operations," Physical Review A (Atomic, Molecular, and Optical Physics), vol. 73, no. 4, p. 042301, 2006.

[27] X.-F. Zhou, Y.-S. Zhang, and G.-S. Guo, "Unitary transformations can be distinguished locally," Physical Review Letters, vol. 99, no. 17, p. 170401, 2007.

[28] R. Y. Duan, Y. Feng, and M. S. Ying, "Entanglement is not necessary for perfect discrimination between unitary operations," Physical Review Letters, vol. 98, no. 10, p. 100503, 2007.

[29] , "Local distinguishability of multipartite unitary operations," Physical Review Letters, vol. 100, no. 2, p. 020503, 2008.

[30] A. Acín, "Statistical distinguishability between unitary operations," Phys. Rev. Lett., vol. 87, no. 17, p. 177901, Oct 2001.

[31] S. Mozes, J. Oppenheim, and B. Reznik, "Deterministic dense coding with partially entangled states," Phys. Rev. A (Atomic, Molecular, and Optical Physics), vol. 71, no. 1, p. 012311, 2005.

[32] M. Artin, Alegbra. Prentice Hall; United States Ed edition, 1991.

[33] R. Hartshorne, Alegbraic Geometry. Springer-Verlag New York, 1977.

[34] I. R. Shafarevich, Basic Algebraic Geometry 1: Varieties in Projective Space. Springer-Verlag, Berlin, second ed, 1995.

[35] H. Chen, "Quantum entanglement and geometry of determinantal varieties," Journal of Mathematical Physics, vol. 47, no. 5, p. 052101, 2006.

[36] T. Cubitt, A. Montanaro, and A. Winter, "On the dimension of subspaces with bounded schmidt rank," Journal of Mathematical Physics, vol. 49, no. 2, p. 022107, 2008.

[37] J. Harris, Alegbraic Geometry, A First Course. Springer Berlin, 1992. 